\title{
Research Paper: Altered Neural Response Induced by Central-Fatigue in the Cortical Area During High-Intensity Interval Pedaling
}

\author{
Mehrangiz Ghorbani $^{1,2}$, Farshad Ghazalian ${ }^{1^{*}}$ (D), Khosrow Ebrahim ${ }^{3}$, Hossein Abednatanzi ${ }^{1}$ \\ 1. Department of Physical Education and Sport Sciences, Faculty of Humanities and Social Sciences, Science and Research Branch, Islamic Azad \\ University, Tehran, Iran. \\ 2. Department of Physical Education and Sport Sciences, Bijar Branch, Islamic Azad University, Bijar, Iran. \\ 3. Department of Physical Education and Sport Sciences, Faculty of Humanities, Shahid Beheshti University, Tehran, Iran.
}

\begin{tabular}{|c|c|}
\hline $\begin{array}{l}\text { Use your device to scan } \\
\text { and read the article online }\end{array}$ & \\
\hline 口irtis & $\begin{array}{l}\text { Fatigue in the Cortical Area During High-Intensity. Basic and Clinical Neuroscience, 10(6), 631-640. https://doi.org/10.32598/ } \\
\text { BCN.9.10.440 }\end{array}$ \\
\hline 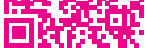 & doli https://doi.org/10.32598/BCN.9.10.440 \\
\hline
\end{tabular}

(c) (i) (\$)

Article info:

Received: 22 Dec 2018

First Revision: 10 Jan 2019

Accepted: 30 Jun 2019

Available Online: 01 Nov 2019

Keywords:

Fatigue,

Electroencephalography, Highintensity interval training

\section{A B S T RA C T}

Introduction: The central-governor model explains the mechanism of endurance exerciseinduced central fatigue, but high-intensity exercise-induced central fatigue has not been investigated yet. This study aimed to research how central fatigue during high-intensity intermittent pedaling alters the neural response, which results in Electroencephalography (EEG) recordings.

Methods: We assessed neural response by measuring the alternation of brainwave spectral power during an intermittent high-intensity 60 -minute exercise on an ergometer cycle. The cadences were changed every 10 minutes according to intermittent pattern altering (90-12060-120-60-90 rpm). EEG was used to analyze altering brain function. Heart Rate (HR), Blood Lactate (BL), and Rating of Perceived Exertion (RPE) were measured after the change in cadences.

Results: HR, BL, and RPE increased at a cadence of $120 \mathrm{rpm}$ compared with $60 \mathrm{rpm}$ on the ergometer cycle. The spectral power of EEG, according to cadence $\times$ brainwaves, significantly increased $(\mathrm{P}<0.01)$ in the alpha and beta frequency ranges with a change in cadences between $60 \mathrm{rpm}$ and $120 \mathrm{rpm}$. The spectral power of the EEG significantly increased $(\mathrm{P}<0.01)$ over the whole frequency range from rest to warming (theta: $251 \%$, alpha: $165 \%$, beta: $145 \%$ ) and significantly reduced in theta, alpha, and beta (theta: $176 \%$, alpha: $142 \%$, beta: $77 \%)(\mathrm{P} \leq 0.01)$.

Conclusion: High-intensity exercises (90 and 120 cadences) increased brain function, regardless of fatigue occurrence. High-intensity interval training (HIIT) led to altering the neural response. It would be required to investigate the usefulness of HIIT to treat some of the psychotic disorders.

\section{* Corresponding Author:

Address: Department of Physical Education and Sport Sciences, Faculty of Humanities and Social Sciences, Science and Research Branch, Islamic Azad University, Tehran, Iran.

Tel: +98 (21) 44865011

E-mail:phdghazalian@gmail.com 


\section{Highlights}

- Central nervous system control in cardiac function largely depends on the intensity of training determined by a change of cadence in the ergometer cycle.

- High-intensity interval training increases brain neural efficiency.

- Exercise at high intensities improves metabolic function, cardiac function, and breathing capacity.

- The brain's role with attention to its neural response in changing position is crucial. We could emphasize it by planning appropriate training to improve the function of the brain.

\section{Plain Language Summary}

The human brain is a complex part of the body that defines us as human. Sport and exercise are not food but feed the brain and other organs. The brain adjusts for varying commands by changing its functional and structural characteristics, which result in learning and acquiring new skills (in this article, the function is the high-intensity interval pedaling). We review the variety of brain activities induced by fatigue because of two main causes; 1 . local muscular fatigue and 2. mental (central) fatigue. While the local muscular system can recover, the fatigue of the central nervous system can take much longer. High-intensity interval training has the largest impact on the central nervous and cognitive systems. These effects are interpreted as their behavioral outcomes.

\section{Introduction}

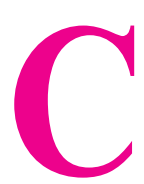

entral fatigue is an unfrequented phenomenon expressed in both populations of healthy (during physical activity) and nonhealthy people. In healthy people, fatigue is a predicted mechanism after a prolonged and intensive activity that is recovered by rest (Gandevia, 2001; Kluger, Krupp, \& Enoka, 2013). Fatigue is experienced in both physical and perceptual aspects (Kluger et al., 2013; Enoka \& Stuart, 1992; Taylor \& Gandevia, 2008). "Central" and "peripheral" fatigue should also be differentiated. Central fatigue is known as the failure of the performance because of the improper function of the central nervous system. Prolonged or intensive exercise leads to central fatigue (Weavil, Sidhu, Mangum, Richardson, \& Amann, 2016). High-intensity interval training (HIIT) improves function and mental health in patients with cardiopulmonary diseases by increasing brain-derived neurotrophic factor and improving neural plasticity in the rats (Luo et al., 2019). Neural plasticity is an expected property of neurons that is vital in adaptation to new situations (Assenza, 2015).

Many researchers have already investigated physical and physiological adaptations, especially the cardiovascular and respiratory systems (Weavil et al., 2016; Taylor, Amann, Duchateau, Meeusen, \& Rice, 2016). Vast research has been organized on the ergometer cycle performance from recreation to the expert levels in elite athletes, especially among endurance athletes (Faria, Parker, \& Faria, 2005). However, changes in the central nervous system and brain activity, as well as changes in the behavioral pattern of neurons during exercise were not considered during high-intensity interval exercises. HIIT could be used to raise sports performance and brain function in men and women alike (Coetsee \& Terblanche, 2017; Chun Kao, Westfall, Soneson, Gurd, \& Hillman, 2017). Brain activity regulates the homeostasis in athletes to delay fatigue and achieve optimal performance during endurance competitions (Noakes, 2011; Skorski \& Abbiss, 2017), but recovery strategies in HIIT induce fatigue and involve organisms rather than modulating homeostasis. Active recovery increased the aspects of central rather than muscle recovery (Rattray, Argus, Martin, Northey, \& Driller, 2015; Giboin, Amiri, Bertschinger, \& Gruber, 2018; Gruet et al., 2014). The study of brain activity by using electroencephalography (EEG) recording has many advantages in sports and training (Skorski \& Abbiss, 2017). All of our thoughts, emotions, and behaviors are due to the communication between neurons within our brains. Brainwaves are produced by synchronized electrical pulses from masses of neurons communicating with each other. Neural response patterns in brain activity in a high-intensity intermittent protocol, which is useful to increase cardiac function, provides useful information to different parts 
of the brain to obtain the general idea of treating many diseases (Nobrega et al., 2014).

Many scientists have investigated the changes in brain activity caused by cycling (Comani et al., 2013). Brain activity is changed by altering functional properties in response to demands, using the central governor model and the homeostasis phenomenon (Inzlicht \& Marcora, 2016; Noakes, 1997; Noakes, 2000; Noakes, 2012). The evaluation of the patterns of neural response of the cerebral cortex (neuroplasticity) (Budde, Wegner, Soya, Rehage, \& McMorris, 2016) during intervals with fatigue contributes to a new insight into functional connectivity between different parts of brain activity in HIIT (Taylor \& Gandevia, 2008; Taylor, et al., 2016; Comani et al., 2013; Gotshall, Bauer, \& Fahrner, 1996). We hypothesized in the present study that neural response patterns induced by the central fatigue in the cortical area are changed by increasing the intensity of training. Therefore, this research aimed to evaluate the effects of central fatigue during high-intensity pedaling with intermittent changes on brain activity. These changes are done by altering functional properties, which results in spectral power frequencies of brainwaves.

\section{Methods}

\subsection{Study Participants}

Fourteen active sprint cyclist women (with the Mean \pm SD age of $25.9 \pm 3.8$ years, height of $170 \pm 1.6 \mathrm{~cm}$, and weight of $62.4 \pm 2.2 \mathrm{~kg}$ ) were selected according to the following inclusion criterion of 4-6 h or 200-240 km $(15-40 \mathrm{~km} / \mathrm{h})$ training background on the flat surface per week (Ludyga, Gronwald, \& Hottenrott, 2015). After the researcher explained the study design, potential risks, and benefits, each participant signed the informed consent form to participate in the study. The exclusion criteria included any cardiovascular and respiratory, metabolic, psychiatric diseases, or orthopedic trauma that could restrict the training. The participants were informed that they were free to leave the study whenever they want. The Ethics Committee of the Medical Faculty of the Azad University of Science and Research Branch of Tehran confirmed this study under the number of IR.IAU.PS.REC. 1397-115, according to the Declaration of Helsinki.

\subsection{Testing protocols}

To determine the anaerobic capacity of the participants, they accomplished the following high-intensity performance test on an ergometer cycle (Monark 894E, An- aerobic Wingate testing): start, $110 \mathrm{~W}$; increase, $10 \mathrm{~W}$; and duration, 4 minutes. The test evaluated anaerobic capacity by a spirometry system (cortex, Meta max 3b, Germany). The participants executed this stage of the test to the failure of power output, and voluntary fatigue occurred. After completing the test, the maximum oxygen consumption $\left(\mathrm{VO} 2_{\text {max }}\right)$ and maximum load $\left(\mathrm{P}_{\max }\right)$ are determined. Aerobic and anaerobic blood lactate (BL), as well as maximum steady-state lactate concentration threshold ([mmol. $\left.\mathrm{l}^{-1}\right]$. [Watt $]^{-1}$ ) were assessed (Mann, Lamberts, \& Lambert, 2013). BL was measured by an enzymatic-amperometric method (Lin, Chen, \& Lin, 2018) in $10 \mu \mathrm{L}$ blood sampled from the earlobe and was analyzed via WinLactat 4.1.0.1 (XE Version, German).

After a week and 3 hours, a meal, the participants performed a 60-minute pedaling test in different cadences with $90 \% \mathrm{VO} 2_{\max }$. The staging test included standard warm-up (10 minutes with $100 \mathrm{~W})$, the main protocol (6 ten-minute steps), and cool-down (10 minutes at 100 W). The cadence intermittent (90-120-60-120-60-90$60 \mathrm{rpm}$ ) changed every 10 minutes during the exercise. The rating of perceived exertion (RPE) was used to assess the intensity of the training interval, using the Borg scale (Borg, 1998). The HR on EEG setup, BL, and RPE were recorded at the end of each 10 minutes. The rest were recorded after the warm-up, after every 10-minute cadence, and after the cool-down (Amann, 2011; Gronf wald, Hoos, Ludyga, \& Hottenrottd, 2018).

\subsection{EEG recordings and analysis}

Electroencephalography (64-Channel QuickAmpEEG system, Brain Products, Germany) was used in continuous brain cortical activity recording. The changes in brainwave signals were symmetrically recorded by 21 active surface electrodes (Brain Products, EasyCap, Germany; Fp1, Fp2, F7, F8, F3, Fz, F4, FC7, FC8, T7, T8, C3, CZ, C4, CP1, P7, P3, P4, PZ, P8, CP2, 10:20 Fixed EEG flexible caps [EasyCAP, Germany]) (Klem, Lüders, Jasper, \& Elger, 1999; Jung, Makeig, Bell, \& Sejnowski, 1998). We implemented the active $\mathrm{Ag} / \mathrm{AgCl}$ electrodes in an active circuitry, ActiShield, a system that allowed the recording of high contact resistance to $60 \mathrm{k} \Omega$. The electrode impedances were controlled by EasyCAP Drivers 6.10.70.001 Software version 1.2.5.3 (Brain Products, Germany), the signals were recorded by Brain Vision Recorder 1.03 (Brain Products, Germany), and the data were sampled at a frequency of $500 \mathrm{~Hz}$. Matlab EEGLAB software was used for offline processing EEG raw data. EEG data were analyzed at rest, after warmup, before each change in cadence, after cool-down, rest, and exercise. After editing, the data were filtered: high- 
pass, $1.5 \mathrm{~Hz}$, octave ${ }^{-1} 12 \mathrm{~dB}$ slope; low-pass, $50 \mathrm{~Hz}$, octave $^{-1} 48 \mathrm{~dB}$ between $1-2 \mathrm{~Hz}$ and $>50 \mathrm{~Hz}$ for removing muscle artifacts. Artifacts up to theta frequencies $(4 \mathrm{~Hz})$ and minimum frequencies of beta $(30 \mathrm{~Hz})$ were eliminated by frequency analysis and, then, a manual raw data inspection was carried out to identify the artifacts. Also, independent components' analysis was calculated to remove artifacts from the main signals (Faria et al, 2005; Faria, Parker, \& Faria, 2005). This control was fixed offline and analyzed only by EEG sections without artifacts. After the edited markers, the signals were divided into 4 S-data-sets with a corrected baseline (Amann, 2011) at the last minute of each time point. Each participant and measuring time point 5 artifact-free segments were analyzed by the Fast-Fourier transform method (Maximum resolution, power in $\mu \mathrm{V} 2$, full-range use, hanging window, window length: $20 \%$ ). The received frequency spectrum was divided into 3 frequencies: theta, 4-7 Hz; alpha, 8-15 Hz; and beta, 16-30 Hz. For further processing, the values were transferred to Microsoft Excel 2016. The analysis of 21 electrodes for each frequency range and time point were measured. The absolute values of spectral power as some changes from individual resting conditions $(100 \%)$ was calculated to extract the effect of the different base EEG types (Faria et al, 2005).

\subsection{Statistical analysis}

The obtained data were analyzed in SPSS V. 24. The Kolmogorov-Smirnov test was performed to evaluate the normal distribution of data. In the case of a normal distribution, the difference between the time points (spectral power of EEG [\%], HR [ $\left.\mathrm{min}^{-1}\right]$, BL [mmol. $1^{-1}$ ) from analysis of variance test for repeated measurements, cadence $\times$ waves, and cadence $\times$ variables (of HR, BL) were calculated. For ordinal data (RPE), the Wilcoxon test was used. Different points of statistical significance were found out at $\mathrm{P} \leq 0.01(* *)$.

\section{Results}

The participants achieved a Mean $\pm \mathrm{SD} \mathrm{P}_{\mathrm{ma}} \mathrm{x}$ of $308.92 \pm 20.61(90 \%$ of the anaerobic lactate threshold: $224.71 \pm 19.00 \mathrm{~W})$ and the $\mathrm{VO} 2$ of $40.43 \pm 3.67 \mathrm{~mL}$. $\mathrm{min}^{-1} \cdot \mathrm{kg}^{-1}$ in ergometer cycle. The values of the heart rate $(\mathrm{HR})$ and the BL during warm-up and at the beginning of the exercise test significantly increased $(\mathrm{P}<0.01)$. RPE significantly increased $(\mathrm{P}<0.01)$ at the beginning of the exercise test compared to the warm-up period. At $120 \mathrm{rpm}, \mathrm{HR}, \mathrm{BL}$, and RPE were significantly higher than $60 \mathrm{rpm}$. All physiological values significantly decreased $(\mathrm{P}<0.01)$ during the cool-down stage (Table 1, Figure 1 and Figure 2).

The spectral EEG power was significant $(\mathrm{P}<0.01)$ for the entire rest to warm-up frequency ranges (theta: $+251 \%$, alpha: $+182 \%$, beta: $+131 \%$ ). The cadence changes are reflected in the spectral power of the EEG (Table 2 and Figure 1). By comparing the different ca-

Table 1. Rating of perceived exertion, blood lactate, and heart rate during warm-up, 6 states of changing cadences, and cool-down

\begin{tabular}{ccccc}
\hline \multirow{2}{*}{ Pedaling States } & \multicolumn{3}{c}{ Variables } \\
\cline { 2 - 4 } & Cadence $(\mathrm{rpm})$ & RPE (6-20) & Blood Lactate (mmol.I-1) & Heart Rate $\left(\mathbf{m i n}^{-1}\right)$ \\
\hline Warm-up & 90 & $9.25 \pm 1.97$ & $0.8 \pm 0.4$ & $118 \pm 8.67^{* *}$ \\
& 90 & $13.05 \pm 1.17^{* *}$ & $1.5 \pm 0.57^{* *}$ & $144.5 \pm 9.69^{* *}$ \\
$\begin{array}{c}\text { Workload } \\
90 \text { \% of the LT }\end{array}$ & 120 & $15.85 \pm 1.17^{* *}$ & $3.5 \pm 1.12^{* *}$ & $163.81 \pm 9.85^{* *}$ \\
& 60 & $13.74 \pm 1.48^{* *}$ & $1.57 \pm 0.57^{* *}$ & $150.25 \pm 10.47^{* *}$ \\
& 120 & $16.50 \pm 1.79^{* *}$ & $3.5 \pm 1.16^{* *}$ & $168.85 \pm 10.74^{* *}$ \\
& 60 & $14.35 \pm 1.58^{* *}$ & $1.8 \pm 0.45^{* *}$ & $154.58 \pm 10.67^{* *}$ \\
Cool-down & 90 & $14.25 \pm 1.37$ & $1.75 \pm 0.57$ & $157.55 \pm 11.71^{* *}$ \\
\hline
\end{tabular}

NEUR:SCIENCE

SD. Standard Deviation; RPE. Rating of Perceived Exertion; RPM. Revolutions Per Minute; BL. Blood Lactate; HR. Heart Rate Data are presented as Mean \pm SD. ${ }^{*}$ Significant levels are at $\mathrm{P}<0.01$. 
Table 2. Variables of electroencephalography spectral power in rest, warm-up, 6 states of changing cadences, cool-down and, and rest

\begin{tabular}{ccccc}
\hline \multirow{2}{*}{ Pedaling States } & \multicolumn{3}{c}{ Variables } & Alpha (\%) \\
\cline { 2 - 4 } & Cadence $(\mathrm{rpm})$ & Theta $(\%)$ & 100 & 100 \\
\hline Rest & - & 100 & $230 \pm 130^{* *}$ & $282 \pm 200^{* *}$ \\
Warm-up & 90 & $350 \pm 160^{* *}$ & $230 \pm 110$ & $308 \pm 284$ \\
& 90 & $345 \pm 194$ & $255 \pm 145$ & $361 \pm 395$ \\
Workload at & 120 & $317 \pm 201$ & $145 \pm 82^{* *}$ & $171 \pm 112^{* *}$ \\
90 of the LT & 60 & $200 \pm 133^{* *}$ & $210 \pm 166$ & $335 \pm 455$ \\
& 120 & $260 \pm 175$ & $112 \pm 57^{* *}$ & $143 \pm 80$ \\
& 60 & $165 \pm 90^{* *}$ & $152 \pm 111^{* *}$ & $150 \pm 85$ \\
\hline
\end{tabular}

RPM. Revolutions Per Minute. **Significant levels are at $\mathrm{P}<0.01$. All data are shown via Mean \pm SD.

dences, the spectral power of the EEG $(\mathrm{P}<0.01)$, in theta, beta. The brain activity at the resting stage significantly decreased (theta: $-67 \%$, alpha: $-58 \%$, beta: $-63 \%$ ) in all cadences $(\mathrm{P}<0.01)$ compared with the beginning $(90$ rpm). According to the EEG brain map in the cadence of $120 \mathrm{rpm}$ theta-waves, clear in all the brain (Figure 3).

\section{Discussion}

The brain neural response decreases in a fatigue condition during pedaling. EEG recordings in different intensi- ties, but at the same workload, showed a higher cadence pedaling frequency, associated with increased brain activity, HR, BL, and RPE. When recruiting muscle fibers increases, muscular coordination and higher cardiovascular function are needed to maintain a higher cadence (Enoka \& Stuart, 1992; Taylor \& Gandevia, 2008; Del Percio et al., 2009). BL increased in the state of high cadence $(120 \mathrm{rpm})$ and decreased at a low cadence (60 $\mathrm{rpm})$. These results support past results on how the training's intensity changes affect the energy supply (Del Percio et al., 2009; CE, Rampichini, \& Veicsteinas, 2009).

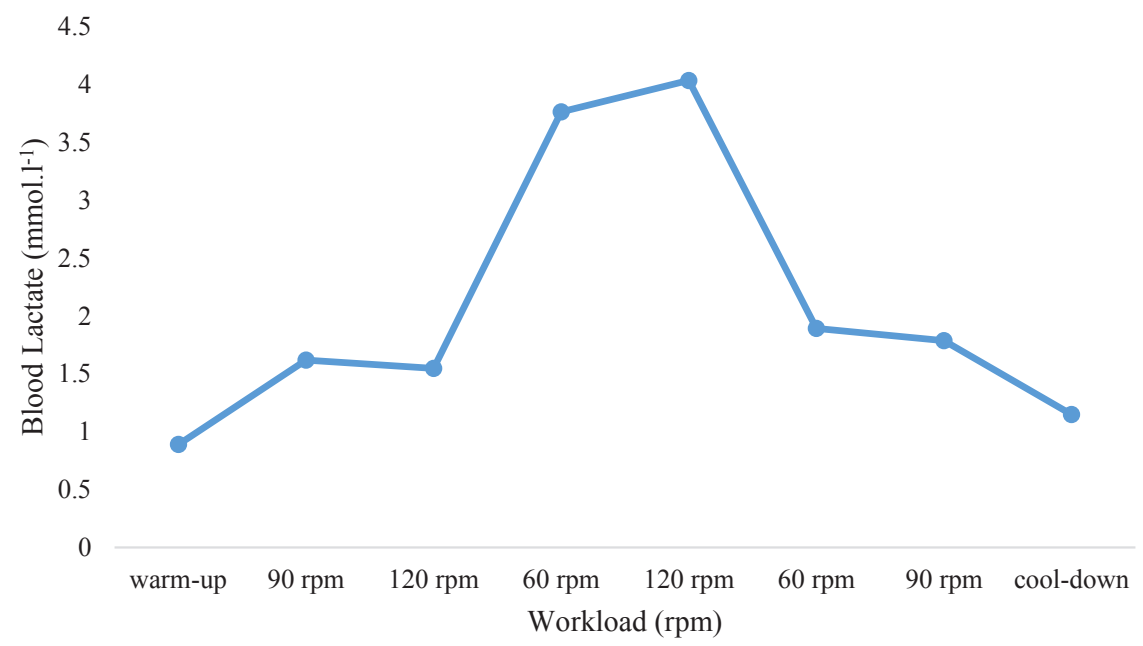

NEUR SCIENCE

Figure 1. Blood lactate (mmol. $\mathrm{l}^{-1}$ ) variability during warm-up, 6 states of changing cadences, and cool-down 


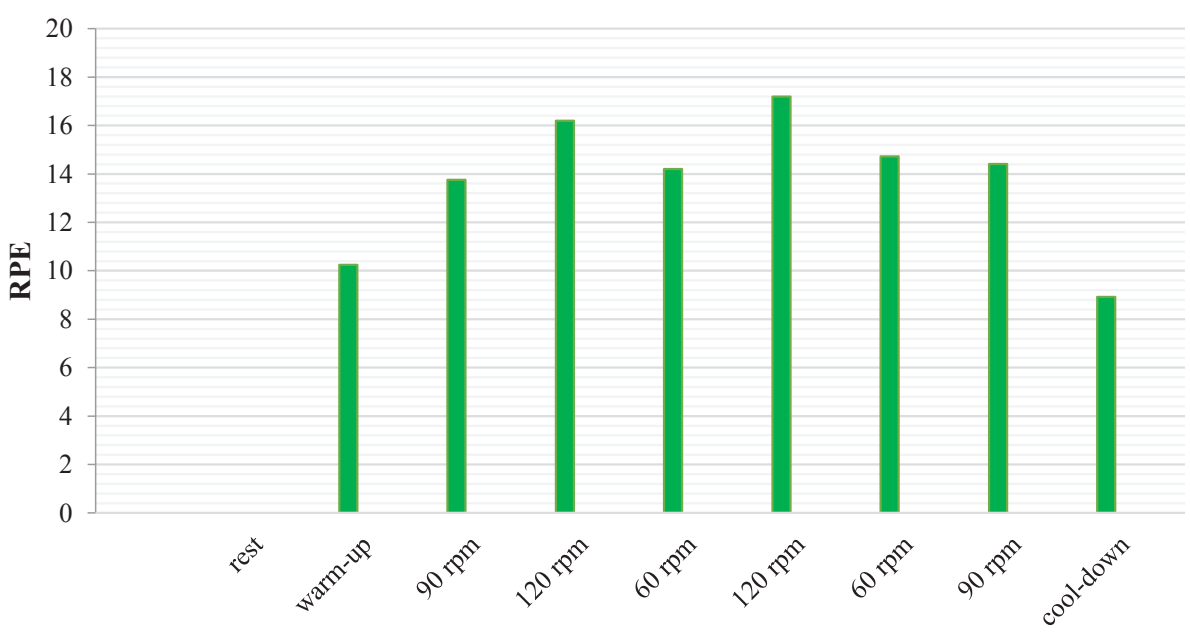

Work Load (rpm)

NEUROSCIENCE

Figure 2. Rating of perceived exertion variability during warm-up, 6 states of changing cadences, and cool-down

In a steady-state workload, BL rises further at cadences from $100 \mathrm{rpm}$ to $120 \mathrm{rpm}$ (CE, et al., 2009) because of the enhanced HR caused by enhancing the cadence. Some investigators suggest that blood flow and, therefore, BL accumulation increases in the involved muscles (CE, et al., 2009). The consciousness of higher cadence exertion enhances with an increased HR and BL accumulation (CE, et al, 2009; Del Percio et al., 2009). Bailey showed a correlation between brain activity oscillations and physiological aspects of performance (Bailey, Hall, Folger, \& Miller, 2008). The power spectral increase of the EEG has been clear in $120 \mathrm{rpm}$ intensity. The brain activity should adapt to the performance intensity and power output in sports. Fatigue reduces brain activity. (Noakes, 2011; Weavil et al., 2016) also showed that brainwave oscillations reduced after a prolonged exhaus- tive exercise protocol displayed in the theta and alpha frequencies. Gronwald et al., performed the same research in cardiovascular regulations in exercise-induced fatigue by emphasizing the changes of cadences (Gronwald et al., 2018). The neural efficiency hypothesis was approved concerning the adaptation of the brain with the frequency of cycling with a lower level of beta activity in cyclists (Ludyga, Gronwald, \& Hottenrottd, 2016). Ludyga also showed a reduction in brain activity after intensive ergometer cycling training. It was approved as a mechanism of fatigue to sustain homeostasis, which was explained in the central governor theory. To describe the fatigue mechanism, Noakes has proposed the central governor model for over two decades. This model introduces the unconscious mind of the brain in regulating the power output by modulating the force and ap-

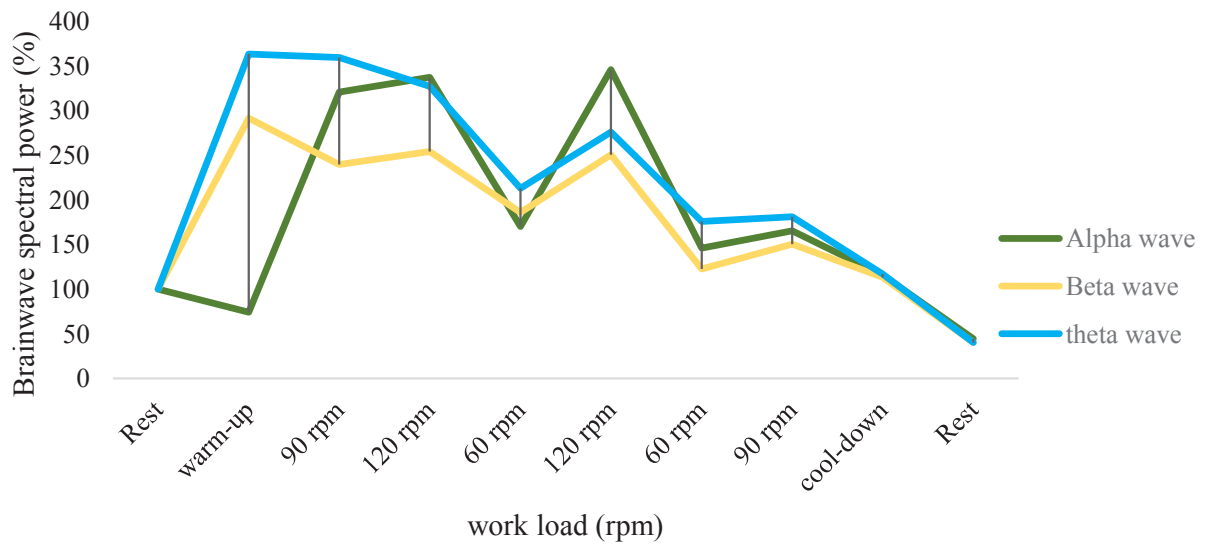

Figure 3. Percent variation in spectral power calculated over 21 electrodes compared to the rest situation (100\%) and averaged over the full electroencephalography frequency dominant $(4-30 \mathrm{~Hz})$ of participants 
plying the motor units to maintain homeostasis, which keeps the body from disastrous physiological disorders. In contrast, neural efficacy assumption depends on task demand and conscious awareness. The better adaptation of the brain activation product is because of the face to the challenges (Noakes, 2012). The neural efficiency hypothesis was characterized by adapting brain activation with functions. There is a correlation between high-cardiac output and increased BL accumulation during high intensities and, then, the perception of force exertion increased (Hagberg, Mullin, Giese, \& Spitznagel, 1981).

Referring to the results, the variables such as BL and HR altered with the intermittent changes. Both central governor theory and the neural efficiency hypothesis are clear with the findings of this study. Meanwhile, any change in cadence results in significant changes in all variables, and homeostasis maintains according to the central governor model. These changes occurred because they were adaptive and changed by the constant workload. A more complex cognitive task is consistent with this task; so, carrying out to a long, intense exercise is needed to reduce the energy reserves of the individual (Inzlicht \& Marcora, 2016). It was mentioned as self-control or inhibitory control, a term borrowed from psychology. Self-control is the ability to regulate one's body requirements, emotions, thoughts, and behavior in the face of internal or external stimulations to achieve specific goals (DeLisi, 2014).

The beta brainwave is a fast activity; it is present when we are attentive, alert, engaged in decision-making, judgment, problem-solving, or focused on mental activity. This band was presented in the frontal, occipital, and temporal lobe in $120 \mathrm{rpm}$. The frontal lobe includes dopamine neurons. The dopaminergic pathways are related to attention, motivation, reward, planning, and short-run memory duties. Dopamine limits sensory information that arrived from the thalamus to the forebrain. The motivation was considered in sports and exercise. The theta brain state, where it is realized, everyone can create everything and change reality immediately. The theta band is displayed in the entire area of the brain in approximately $120 \mathrm{rpm}$. Theta band is powerful and should be considered the subconscious (mind condition between the conscious and the unconscious).

Fatigue induced by high-intensity intermittent pedaling disables the brain, but at high intensities (cadence of 120 $\mathrm{rpm}$ ) were obtained at one session, with repeatability in the 8 interval steps of the high-intensity workload following a behavioral pattern. The results were obtained at one session, with repeatability in the 8 interval steps of the high-intensity workload following a behavioral pattern. With the onset of fatigue, the activity of the brain decreases, but with increasing intensity, the activity of the brain increases. Fatigue in relation to HIIT creates new behavioral patterns in response to various situations according to the spectral power of alpha, beta, and theta with attention to involving areas of the cortical brain.

\section{Conclusion}

Thus, the pattern of brain activity oscillations depends on exercise intensity, and individually-preferred cadence contributes to the positive response of the cognitive process (Brümmer, Schneider, Strüder, \& Askew, 2011). The activity of the brain cortex is crucial to achieving performance and power output in exercises. The higher parts of the cortex process cognitive abilities and are responsible for tolerating pain and delaying the pleasure by self-control. HIIT improves cognitive processes in older people (Coetsee \& Terblanche, 2017; Samuel et al., 2017). In conclusion, fatigue induced by high-intensity intermittent training leads to a strategy to maintain homeostasis, not a mechanism to survive to the extent that is mentioned by the central governor model.

\section{Ethical Considerations}

\section{Compliance with ethical guidelines}

All procedures were done following the Helsinki Declaration. The Ethics Committee of the Medical Faculty of Azad University of Science and Research Branch, Tehran (1397-115), approved the present research.

\section{Funding}

This research did not receive any funding.

\section{Authors' contributions}

Designed and performed experiments, analyzed data and co-wrote the paper, performed bioinformatics analyses, Performed experiments: Mehrangiz Ghorbani; Supervisor, Performed transporter experiments: Farshad ghazzalian; Coadvisor, performed metabolomics analyses: Kosrow Ebrahim and Hossein Abed natanzi.

\section{Conflict of interest}

The authors declared no conflict of interest. 


\section{References}

Amann, M. (2011). Central and peripheral fatigue: Interaction during cycling exercise in humans. Medicine $\mathcal{E}$ Science in Sports $\mathcal{E}$ Exercise, 43(11), 2039-45. [DOI:10.1249/ MSS.0b013e31821f59ab] [PMID]

Assenza, G. (2015). A useful Electroencephalography (EEG) marker of brain plasticity: Delta waves. Neural Regeneration Research, 10(8), 1216-7. [DOI:10.4103/1673-5374.162698] [PMID] [PMCID

Bailey, S., Hall, E., Folger, S., \& Miller, P. (2008). Changes in EEG during graded exercise on a recumbent cycle ergometer. Journal of Sports Science \& Medicine, 7(4), 505-11. 01373 [DOI:10.1097/00005768-200405001-01373]

Borg, G. (1998). Borg's perceived exertion and pain scales. Champaign, Illinois: Champaign.

Brümmer, V., Schneider, S., Strüder, H., \& Askew, C. (2011). Primary motor cortex activity is elevated with incremental exercise intensity. Neuroscience, 181(1), 150-62. [DOI:10.1016/j. neuroscience.2011.02.006] [PMID]

Budde, H., Wegner, M., Soya, H., Rehage, C., \& McMorris, T (2016). Neuroscience of Exercise: Neuroplasticity and Its Behavioral Consequences. Neural Plasticity, 2016, 3643879. [DOI:10.1155/2016/3643879] [PMID] [PMCID]

CE, E., Rampichini, S., \& Veicsteinas, A. (2009). Effects of tiredness on visuospatial attention processes in élite karate athletes and non-athletes. Physiology, 147, 1-10. [PMID]

Chun Kao, S., Westfall, D., Soneson, J., Gurd, B., \& Hillman, C. (2017). Comparison of the acute effects of high-intensity interval training and continuous aerobic walking on inhibitory control. Psychophysiology, 54(9), 1335-45. [DOI:10.1111/ psyp.12889] [PMID]

Coetsee, C., \& Terblanche, E. (2017). The effect of three different exercise training modalities on cognitive and physical function in a healthy older population. European Review of Aging and Physical Activity, 14(13), 1-10. [DOI:10.1186/s11556-0170183-5] [PMID] [PMCID]

Comani, S., di Fronso, S., Filho, E., Castronovo, M., Schmid, M. Bortoli, L., et al., (2013). Attentional focus and functional connectivity in cycling: An EEG case study. Springer, 41(1), $137-$ 40. [DOI:10.1007/978-3-319-00846-2_34]

Del Percio , C., Babiloni , C., Infarinato, F., Marzano , N., Iacoboni , M., Lizio , R., et al., (2009). Effects of tiredness on visuospatial attention processes in élite karate athletes and nonathletes. Italian Archives of Biology, 147(1-2), 1-10. [PMID]

DeLisi, M. (2014). Chapter 10: Low self-control is a brain-based disorder. In, K. M. Beaver, J. C. Barnes, \& B. B. Boutwell (Eds.), The nurture versus biosocial debate in criminology: On the origins of criminal behavior and criminality (pp. 172-184). Thousand Oaks, California: Sage Publications. [DOI:10.4135/9781483349114]

Enoka, R., \& Stuart, D. (1992). Neurobiology of muscle fatigue Journal of Applied Physiology, 72(5), 1631-48. [DOI:10.1152/ jappl.1992.72.5.1631] [PMID]

Faria, E., Parker, D., \& Faria, I. (2005). The science of cycling: Factors affecting performance - Part 2. Sports Medicine, 35(4), 313-37. [DOI:10.2165/00007256-200535040-00003] [PMID]
Faria, E., Parker, D., \& Faria, I. (2005). The science of cycling: Physiology and training - Part 1. Sports Medicine, 35(4), 285312. [DOI:10.2165/00007256-200535040-00002] [PMID]

Gandevia, S. (2001). Spinal and supra spinal factors in human muscle fatigue. Physiological Reviews, 81(4), 1725-89. [DOI:10.1152/physrev.2001.81.4.1725] [PMID]

Giboin, L., Amiri, E., Bertschinger, R., \& Gruber, M. (2018). Active recovery affects the recovery of the corticospinal system but not of muscle contractile properties. PLoS One, 13(5), 1-16. [DOI:10.1371/journal.pone.0197339] [PMID] [PMCID]

Gotshall, R., Bauer, T., \& Fahrner, S. (1996). Cycling cadence alters exercise hemodynamics. International Journal of Sports Medicine, 17(1), 17-21. [DOI:10.1055/s-2007-972802] [PMID]

Gronwald, T., Hoos, O., Ludyga, S., \& Hottenrott, K. (2019) Non-linear dynamics of heart rate variability during incremental cycling exercise. Research in Sports Medicine, 27(1), 88-98. [DOI:10.1080/15438627.2018.1502182] [PMID]

Gruet, M., Temesi, J., Rupp, T., Levy, P., Verges, S., \& Millet, G. (2014). Dynamics of corticospinal changes during and after high-intensity quadriceps exercise. Experimental Physiology 99(8), 1053-64. [DOI:10.1113/expphysiol.2014.078840] [PMID]

Hagberg, J., Mullin, J., Giese, M., \& Spitznagel, E. (1981). Effect of pedaling rate on submaximal exercise responses of competitive cyclists. Journal of Applied Physiology: Respiratory, Environmental and Exercise Physiology, 51(2), 447-51. [DOI:10.1152/jappl.1981.51.2.447] [PMID]

Inzlicht, M., \& Marcora, S. M. (2016). The central governor model of exercise regulation teaches us precious little about the nature of mental fatigue and self-control failure. Frontiers in Psychology, 7, 656. [DOI:10.3389/fpsyg.2016.00656] [PMID] [PMCID]

Jung, T. P., Makeig, S., Bell, A. J., \& Sejnowski, T. J. (1998). In dependent component analysis of electroencephalographic and event-related potential data. In P. F. Poon, \& J. F. Brugge (Eds.), Central Auditory Processing and Neural Modeling (pp. 189-97). Boston: Springer. [DOI:10.1007/978-1-4615-53519_17]

Klem, G. H., Lüders, H. O., Jasper, H. H., \& Elger, C. (1999). The ten-twenty electrode system of the International Federation. Electroencephalography and Clinical Neurophysiology, 52(3), 3-6. [PMID]

Kluger, B., Krupp, L., \& Enoka, R. (2013). Fatigue and fatigability in neurologic illnesses: Proposal for a unified taxonomy. Neurology, 80(4), 409-16. [DOI:10.1212/WNL.0b013e31827f07be] [PMID] [PMCID]

Lin, T., Chen, C., \& Lin, M. (2018). Enzyme-free amperometric method for rapid determination of histamine by using surface oxide regeneration behavior of copper electrode. Sensors and Actuators B: Chemical, 255(3), 2838-43. [DOI:10.1016/j. snb.2017.09.101]

Ludyga, S., Gronwald, T., \& Hottenrott, K. (2015). Do male and female cyclists' cortical activity differ before and during cycling exercise. Journal of Sport and Exercise Psychology, 37(6), 617-25. [DOI:10.1123/jsep.2015-0078] [PMID]

Ludyga, S., Gronwald, T., \& Hottenrott, K. (2016). The athlete's brain: Cross-sectional evidence for neural efficiency during cycling exercise. Neural Plasticity, 7(1), 1-7. [DOI:10.1155/2016/4583674] [PMID] [PMCID] 
Luo, L., Li, C., Deng, Y., Wang, Y., Meng, P., \& Wang, Q. (2019). High-intensity interval training on neuroplasticity, balance between brain-derived neurotrophic factor and precursor brain-derived neurotrophic factor in post stroke depression rats. Journal of Stroke and Cerebrovascular Diseases, 28(3) 672-82. [DOI:10.1016/j.jstrokecerebrovasdis.2018.11.009] [PMID]

Mann, T., Lamberts, R., \& Lambert, M. (2013). Methods of prescribing relative exercise intensity: Physiological and practical considerations. Sports Medicine, 43(7), 613-25. [DOI:10.1007/ s40279-013-0045-x] [PMID]

Noakes, T. D. (1997). Challenging beliefs: Ex Africa semper aliquid novi. Medicine \& Science in Sports \& Exercise, 29(5), 57190. [DOI:10.1097/00005768-199705000-00001] [PMID]

Noakes, T. D. (2000). Physiological models to understand exercise fatigue and the adaptations that predict or enhance athletic performance. Scandinavian Journal of Medicine \& Science in Sports, 10(3), 123-45. [DOI:10.1034/j.16000838.2000.010003123.x] [PMID]

Noakes, T. D. (2011). Is it time to retire the A.V. hill model. Sports Medicine, 41(4), 263-77. [DOI:10.2165/11583950-00000000000000] [PMID]

Noakes, T. D. (2011). Time to move beyond a brainless exercise physiology: The evidence for complex regulation of human exercise performance. Applied Physiology, Nutrition, and Metabolism, 36(1), 23-35. [DOI:10.1139/H10-082] [PMID]

Noakes, T. D. (2012). Fatigue is a brain-derived emotion that regulates the exercise behavior to ensure the protection of whole body homeostasis. Frontiers in Physiology, 3(82), 1-13. [DOI:10.3389/fphys.2012.00082] [PMID] [PMCID]

Noakes, T. D., Peltonen, J., \& Rusko, H. (2001). Evidence that a central governor regulates exercise performance during acute hypoxia and hyperoxia. Journal of Experimental Biology, 204(18), 3225-34. [PMID]

Nobrega, A., O'Leary, D., Silva, B., Marongiu, E., Piepoli, M., \& Crisafulli, A. (2014). Neural regulation of cardiovascular response to exercise: Role of central command and peripheral afferents. BioMed Research International, 2014, 478965. [DOI:10.1155/2014/478965] [PMID] [PMCID]

Rattray, B., Argus, C., Martin, K., Northey, J., \& Driller, M. (2015). Is it time to turn our attention toward central mechanisms for post-exertional recovery strategies and performance? Frontiers in Physiology, 6(79), 1-14. [DOI:10.3389/ fphys.2015.00079] [PMID] [PMCID]

Samuel, R., Zavdy, O., Levav, M., Reuveny, R., Katz, U., \& Dubnov-Raz, G. (2017). The effects of maximal intensity exercise on cognitive performance in children. Journal of Human $\mathrm{Ki}$ netics, 57(1), 85-96. [DOI:10.1515/hukin-2017-0050] [PMID] [PMCID]

Skorski, S., \& Abbiss, C. (2017). The manipulation of pace within endurance sport. Frontiers in Physiology, 8(1), 102-10. [DOI:10.3389/fphys.2017.00102]

Taylor, J., \& Gandevia, S. (2008). A comparison of central aspects of fatigue in submaximal and maximal voluntary contractions. Journal of Applied Physiology, 104(2), 542-50. [DOI:10.1152/japplphysiol.01053.2007] [PMID]

Taylor, J., Amann, M., Duchateau, J., Meeusen, R., \& Rice, C. (2016). Neural contributions to muscle fatigue: From the brain to the muscle and back again. Medicine and Science in Sports and Exercise, 48(11), 2294-306. [DOI:10.1249/ MSS.0000000000000923] [PMID] [PMCID]

Weavil, J. C., Sidhu, S. K., Mangum, T. S., Richardson, R. S., \& Amann, M. (2016). Fatigue diminishes motoneuronal excitability during cycling exercise. Journal of Neurophysiology, 116(4), 1743-51. [DOI:10.1152/jn.00300.2016] [PMID] [PMCID] 
This Page Intentionally Left Blank 